\title{
Revisão de intervenções cognitivas em pacientes com Afasia Progressiva Primária
}

\author{
Review of cognitive interventions in Primary Progressive \\ Aphasia patients
}

\section{Revisión de intervenciones cognitivas en pacientes con Afasia Progresiva Primaria}

\author{
Karin Reuwsaat Vieira ${ }^{1}$, Ariane Cristina Ramello de Carvalho ${ }^{2}$, \\ Eliane Correa Miotto ${ }^{3}$
}

\begin{abstract}
1.Psicóloga, Aluna do curso de Reabilitação Neuropsicológica, Centro de Estudos de Neurologia Prof. ${ }^{\circ}$ Dr. Antonio Branco Lefèvre, Divisão de Clínica Neurológica, Hospital das Clínicas da Faculdade de Medicina da USP, São Paulo-SP, Brasil.

2.Psicóloga, Mestre e doutoranda em Distúrbios do Desenvolvimento, Universidade Presbiteriana Mackenzie, São Paulo-SP, Brasil.

3.Psicóloga, Livre Docente e Orientadora da Pós-Graduação, Departamento de Neurologia da FMUSP. Coordenadora do Curso de Reabilitação Neuropsicológica - Centro de Estudos de Neurologia Prof. ${ }^{\circ}$ Dr. Antonio Branco Lefèvre, Divisão de Clínica Neurológica, Hospital das Clínicas da Faculdade de Medicina da USP, São Paulo-SP, Brasil.
\end{abstract}

\section{Resumo}

Introdução. A afasia progressiva primária (APP) é uma síndrome clínica caracterizada pelo comprometimento da fala e da linguagem decorrente da neurodegeneração das redes neurais. Objetivo. Analisar as técnicas ecológicas de reabilitação neuropsicológica e a sua efetividade no tratamento de indivíduos com APP por meio de uma revisão sistemática de literatura. Método. Foram selecionados, no PubMed, 42 estudos em pacientes com idade entre 50 e 80 anos e com diagnóstico APP, usando os descritores Primary Progressive Aphasia/rehabilitation/therapy publicados entre 2014 e 2020. Destes, foram excluídos os estudos de caso e aqueles com tratamentos medicamentosos ou com estimulação transcraniana. Resultados. Cinco publicações foram selecionadas seguindo os critérios de inclusão e exclusão. Os estudos construíram a base das técnicas de estimulação da nomeação através da apresentação de imagens com seus respectivos nomes. Estudos avançaram em treinar com variações nas imagens de mesmo conteúdo para analisar a generalização da aprendizagem. Conclusão. Dentre os estudos selecionados pode se observar uma melhora significante nos pacientes de APP, tanto com a generalização como na manutenção. Portanto, a intervenção de nomeação de imagens contribui para proteção ou melhora da função linguística em declínio nesse grupo de pacientes. Entretanto, existem poucos estudos com amostragem randomizada e com grupo controle para avaliar os efeitos de reabilitação neuropsicológica de pacientes com APP.

Unitermos. Afasia Progressiva Primária; reabilitação; terapia

\footnotetext{
Abstract

Introduction. Primary progressive aphasia (PPA) is a clinical syndrome characterized by impaired speech and language resulting from neural networks' neurodegeneration. Objective. To analyze ecological neuropsychological rehabilitation techniques and their effectiveness in treating individuals with PPA through a systematic literature review. Method. We selected 42 studies in PubMed in patients aged between 50 and 80 years and diagnosed with PPA, using the keywords Primary Progressive Aphasia/rehabilitation/therapy. Of these, case studies and drug treatments or transcranial stimulation were excluded. Results. We selected five publications according to the inclusion and exclusion criteria. The reviews built the basis for naming stimulation techniques by presenting images with their respective names. Studies have advanced in training with variations in images of the same content to analyze learning generalization. Conclusion. Among the selected studies, a significant improvement was
} 
observed in PPA patients, both with generalization and maintenance. Therefore, the image naming intervention contributes to the protection or advance of the declining linguistic function in this group of patients. However, few studies with randomized sampling and a control group assess the effects of neuropsychological rehabilitation of patients with PPA.

Keywords. Primary Progressive Aphasia; rehabilitation; therapy

\section{Resumen}

Introducción. La afasia progresiva primaria (APP) es un síndrome clínico que se caracteriza por alteraciones del habla y el lenguaje como resultado de la neurodegeneración de las redes neuronales. Objetivo. Analizar las técnicas de rehabilitación neuropsicológica ecológica y su efectividad en el tratamiento de personas con APP a través de una revisión sistemática de la literatura. Método. En PubMed se seleccionaron 42 estudios en pacientes con edades comprendidas entre 50 y 80 años y diagnosticados de APP, utilizando las palabras clave Primary Progressive Aphasia/rehabilitation/therapy. De estos, se excluyeron los estudios de casos y los tratamientos farmacológicos o con estimulación transcraneal. Resultados. Se seleccionaron cinco publicaciones según los criterios de inclusión y exclusión. Los estudios sentaron las bases para nombrar las técnicas de estimulación al presentar imágenes con sus respectivos nombres. Se han avanzado estudios en la formación con variaciones en imágenes de un mismo contenido para analizar la generalización del aprendizaje. Conclusión. Entre los estudios seleccionados, se puede observar una mejora significativa en pacientes con APP, tanto con generalización como con mantenimiento. Por tanto, la intervención de denominación de imágenes contribuye a la protección o mejora de la función lingüística en declive en este grupo de pacientes. Sin embargo, existen pocos estudios con muestreo aleatorizado y con grupo control para evaluar los efectos de la rehabilitación neuropsicológica de pacientes con APP.

Palabras clave. Afasia progresiva primaria; rehabilitación; terapia

Trabalho realizado na Faculdade de Medicina da USP, São Paulo-SP, Brasil.

\section{INTRODUÇÃO}

\section{A afasia progressiva primária (APP) é uma síndrome clínica caracterizada pelo comprometimento da fala e da} linguagem decorrente da neurodegeneração das redes neurais $^{1}$ causado pelo desenvolvimento de proteínas patogênicas ${ }^{2}$. Os critérios de consenso para o diagnóstico de APP têm as seguintes variantes: (1) a variante não fluente/agramática (APPvnf), (2) a variante semântica (APPvs), (3) a variante logopênica (APPvl) e (4) a variante atípica (APPva) ${ }^{3}$.

O diagnóstico diferencial de cada variante de APP vai depender de três algoritmos: da avaliação clínica, de estudos 
de exames de imagens e do diagnóstico clínico realizado ${ }^{4}$. Segundo as diretrizes iniciais propostas por Mesulam ${ }^{5}$, os pacientes devem primeiro atender aos critérios de inclusão e exclusão. São três critérios de inclusão: (1) ter como característica proeminente na avaliação clínica a dificuldade com linguagem, (2) o sintoma inicial é o declínio proeminente na capacidade linguística, o qual deve persistir nas fases seguintes e (3) esse déficit deve ser a principal causa de prejuízos nas atividades diárias. Para os itens de exclusão temos: (1) quando o padrão de prejuízos é melhor explicado por outros distúrbios do sistema nervoso, (2) ou melhor explicado por distúrbio psiquiátrico, (3) apresentar prejuízos na memória episódica, memória visual e visuoperceptual na fase inicial e (4) apresentar distúrbio comportamental na fase inicial e com características proeminentes ${ }^{4}$. Os algoritmos de diferenciação das variantes incluem ainda avaliar as características segundo os principais domínios linguísticos na produção de fala (gramática, fala motora, erros de som e pausas na busca de palavras), repetição, compreensão de uma única palavra e sintaxe, nomeação de confrontos, conhecimento semântico e leitura/ortografia6.

Nos critérios para APP da variante não fluente/agramática, é importante observar um esforço na produção da linguagem e na fala (agramatismo), que consiste em frases curtas e simples e omissões de morfemas gramaticais (por exemplo, palavras de função, inflexões) durante avaliação clínica. No início do quadro, pode ser 
observado um prejuízo no planejamento da articulação (apraxia da fala) $^{4}$ que pode consistir em distorções, deleções, substituições, inserções ou transposições de sons de fala, os quais, geralmente, são perceptíveis pelo paciente. Os déficits de compreensão da sintaxe são evidenciados pelo comprometimento da compreensão da sentença. Esses surgem com manifestações apenas na construção de frases complexas, enquanto a compreensão da palavra única e o significado estão preservados ${ }^{4}$. A diferença na compreensão da sentença entre a variante logopênica e a não-fluente, é que, nessa última, a deficiência é claramente influenciada pela complexidade gramatical da sentença. Alterações patológicas desta variante são de degeneração do lobo frontotemporal com mutações na codificação genética da proteína tau (FTLD-tau) ou, com menor frequência, da degeneração do lobo frontotemporal com TDP-43 positivo $(\text { FTLD-TDP })^{4}$. Os estudos de imagem de ressonância magnética podem apresentar atrofia predominante na região fronto insular posterior esquerda. Os resultados no SPECT ou PET indicam hipoperfusão ou hipometabolismo predominantemente na região fronto insular esquerda ${ }^{4}$.

A variante semântica, ou também chamada de demência semântica ${ }^{7}$, tem como característica o declínio gradual do conhecimento semântico e anomia ${ }^{4}$. A compreensão de uma única palavra é gravemente prejudicada, especialmente para itens de baixa frequência no cotidiano. Dificuldade na compreensão de palavras únicas é geralmente a manifestação mais precoce e evidente de um 
déficit de memória semântica generalizado que causa prejuízos no reconhecimento de objetos e de pessoas $^{8}$, no entanto, não apresenta prejuízos na repetição e na produção da fala (gramática e fala motora) ${ }^{4}$. Os prejuízos semânticos, geralmente, estão presentes na maioria das categorias (por exemplo, ferramentas, animais e pessoas) ${ }^{9}$. Na escrita e na leitura são observados prejuízos de disgrafia e dislexia de superfície 4,10 , que é um distúrbio no processo de reconhecimento da palavra pela rota lexical e uma dificuldade de menor intensidade na realização da leitura em voz alta, pela rota fonológica ${ }^{11}$. No caso da variante semântica, as alterações patológicas mais comuns são do tipo FTLD-TDP ${ }^{12,13}$. Os estudos de imagem de ressonância magnética podem apresentar atrofia predominante no lobo temporal anterior. Os resultados no SPECT ou PET indicam hipoperfusão ou hipometabolismo predominantemente no lobo temporal anterior ${ }^{4}$.

A variante logopênica apresenta déficit de repetição de sentenças e na recuperação de palavras (em fala espontânea e nomeação de confronto). A fala espontânea é caracterizada por ritmo lento, com pausas frequentes devido a problemas significativos na busca de palavras, mas não há agramatismo. Os déficits na produção da fala são, portanto, distintos dos pacientes com a variante não fluente, que também falam de maneira lenta e interrompida, mas marcada por erros de fala motora ou por agramatismo ${ }^{14}$. E, os erros são caracterizados geralmente por dificuldades fonológicas e não pelo conhecimento semântico ${ }^{15}$ como na 
variante semântica. Outras características de diagnóstico incluem parafasias fonológicas na fala e nomeação espontânea. As substituições sonoras que resultam em parafasias fonológicas em pacientes logopênicos geralmente são bem articuladas, sem distorções. Esses pacientes apresentam a compreensão e conhecimento da palavra única, discurso poupado e agramatismo poupado ${ }^{4}$. Os estudos de imagem de ressonância magnética podem apresentar atrofia predominante na região perisilviana posterior esquerda ou parietal. Os resultados no SPECT ou PET indicam hipoperfusão ou hipometabolismo predominantemente na região perisilviana posterior esquerda ou parietal ${ }^{4}$.

A reabilitação neuropsicológica tem como objetivo melhorar a qualidade de vida das pessoas com uma lesão cerebral adquirida, causada por um acidente ou por uma doença, seja estática ou progressiva ${ }^{16}$. A utilização de técnicas ecológicas de reabilitação neuropsicológica, que têm uma maior relevância ou semelhança com mundo "real", permitem além da melhoria das funções cognitivas também a generalização e transferencia de aquisição de habilidades para situações reais ${ }^{17}$.

Atualmente, não há intervenções farmacológicas para melhorar ou proteger a função em declínio em indivíduos com APP ${ }^{18}$. Apenas tratamentos e intervenções cognitivolinguísticas direcionadas principalmente, para a anomia com terapia semântica, por ser característica comum a todas variantes. A literatura, que compreende estudos de um único 
tema e de pequenos grupos ${ }^{18}$, mostra uma melhoria significativa para comportamentos treinados, assim como, outros estudos mostram melhorias generalizadas para itens não treinados ${ }^{19}$.

Uma revisão sistemática evidenciou que a reabilitação neuropsicológica contribui para o tratamento de APP ${ }^{20}$. Um dos pontos observados foi a importância de identificar a variante de APP de cada indivíduo para, então, planejar a reabilitação. Assim, os resultados de generalização ocorrem com mais frequência no APPvnf e na APPvl do que na APPvs. Enquanto, a manutenção tem bons resultados independentemente do subtipo APP. Como consequência da natureza degenerativa do APP, a manutenção das habilidades aprendidas é mais evidente a curto prazo do que a longo prazo, na ausência de prática. A principal intervenção utilizada é treino de nomes de objetos (escrita e no audio), que resultam em ganhos para recuperação das palavras tratadas $^{21}$.

Por outro lado, a revisão sistemática com metanálise identificou melhora no desempenho da linguagem para nomeação quando combinado com as técnicas de estimulação cerebral, por estimulação transcraniana de corrente contínua (ETCC), para itens treinados e não treinados, imediatamente após o treinamento e durante o tratamento 22 .

Nesse artigo, temos como objetivo analisar as técnicas ecológicas de reabilitação neuropsicológica e a sua 
efetividade no tratamento de indivíduos com APP por meio de uma revisão de literatura.

\section{MÉTODO}

Para essa revisão sistemática de literatura, realizou-se uma busca de artigos publicados nas bases de dados PubMed entre outubro de 2014 e novembro 2020 que tivessem acesso livre ao texto completo. Para a busca usou-se os seguintes descritores dos constructos: "Primary Progressive Aphasia", "rehabilitation" e "therapy". Foram encontrados 42 artigos e realizada a leitura dos títulos e resumos, destes foram selecionadas 14 publicações para ler a metodologia e averiguar quais preenchiam integralmente os critérios de inclusão e exclusão.

Os critérios de inclusão utilizados foram: pelo menos um adulto com idade entre 50 e 80 anos com diagnóstico de APP, descrição das intervenções cognitivas, presença de avaliação pré- e pós-intervenção, assim como publicações na língua inglesa e portuguesa. Ao passo que os critérios de exclusão consistiram em: revisão de literatura, estudos de caso, tratamentos farmacológicos e tratamento com estimulação transcraniana.

No final foram selecionadas cinco publicações para revisão sistemática de literatura, os quais encontram-se descritos a seguir e resumidos na Tabela 1. 


\section{RESULTADOS}

\section{Os artigos selecionados de acordo com os critérios de}

\section{inclusão e exclusão estão descritos a seguir e encontram-se}

resumidos nas Tabelas 1 e 2 .

Tabela 1. Resumo dos objetivos, amostra e métodos utilizados nas pesquisas.

\begin{tabular}{|c|c|c|c|}
\hline Autores & Objetivos & Amostra & Método \\
\hline $\begin{array}{l}\text { Beales, } \\
\text { Whitworth e } \\
\text { Panegyres }^{23}\end{array}$ & $\begin{array}{l}\text { Analisar os efeitos de } \\
\text { uma intervenção de } \\
\text { recuperação léxica } \\
\text { auto sinalizada entre } \\
\text { as classes de palavras }\end{array}$ & $\begin{array}{l}\text { Para o estudo } \\
\text { participaram } 4 \\
\text { pacientes, idade entre } \\
52-77 \text { anos, três com } \\
\text { diagnóstico APPvs e } \\
\text { um APPvl. }\end{array}$ & $\begin{array}{l}\text { Foram realizadas } 8 \text { sessões de } \\
\text { terapia quinzenal de } 90 \text { minutos ao } \\
\text { longo de } 4 \text { semanas na casa dos } \\
\text { participantes. A intervenção } \\
\text { desenvolveu-se com estratégia de } \\
\text { recuperação léxica auto sinalizada. }\end{array}$ \\
\hline $\begin{array}{l}\text { Savage, Piguet } \\
\text { e Hodges }\end{array}$ & $\begin{array}{l}\text { Desenvolver um } \\
\text { treinamento cognitivo } \\
\text { on-line para } \\
\text { reaprender os nomes } \\
\text { dos objetos. }\end{array}$ & $\begin{array}{l}\text { A amostra tinha cinco } \\
\text { pacientes com idade } \\
\text { entre } 56 \text { e } 71 \text { anos e } \\
\text { diagnostico APPvs. }\end{array}$ & $\begin{array}{l}\text { A intervenção foi realizada pela } \\
\text { internet com uso de computador dos } \\
\text { participantes com softwares } \\
\text { personalizados. As atividades } \\
\text { envolviam o emparelhamento de } \\
\text { fotografias com seu respectivo rótulo }\end{array}$ \\
\hline $\begin{array}{l}\text { Savage, Piguet } \\
\text { e Hodges }\end{array}$ & $\begin{array}{l}\text { Objetivo era treinar e } \\
\text { monitorar o } \\
\text { desempenho de } \\
\text { nomeação em uma } \\
\text { série de pacientes } \\
\text { APPvs, examinando a } \\
\text { manutenção } \\
\text { independente e } \\
\text { assistida }\end{array}$ & $\begin{array}{l}\text { A amostra foi } \\
\text { composta por } \\
\text { indivíduos de } 50 \text { a } 71 \\
\text { anos, com diagnóstico } \\
\text { APPvs, com padrão } \\
\text { típico de atrofia do } \\
\text { lobo temporal anterior } \\
\text { com perda de volume } \\
\text { maior a direita em } 7 \\
\text { pacientes e à } \\
\text { esquerda em } 2 \\
\text { participantes. }\end{array}$ & $\begin{array}{l}\text { O estudo de linha de base foi } \\
\text { individual com base em } 3 \text { listas de } \\
\text { palavras para cada paciente. Foram } \\
\text { treinados por } 5 \text { semanas cada lista. } \\
\text { Ocorreu uma reavaliação no final de } \\
\text { cada semana de terapia e o } \\
\text { desempenho foi monitorado por mais } \\
6 \text { meses, primeiro em intervalos } \\
\text { semanais (nos primeiros } 1 \text { a } 3 \\
\text { meses) e depois em intervalos } \\
\text { mensais. Após a avaliação de dois } \\
\text { meses, o treinamento foi } \\
\text { reintroduzido o procedimento do } \\
\text { treinamento. }\end{array}$ \\
\hline \multirow[t]{2}{*}{$\begin{array}{l}\text { Hoffman, } \\
\text { Clarke, Jones e } \\
\text { Noonan }{ }^{26}\end{array}$} & $\begin{array}{l}\text { Analisou o efeito de } \\
\text { ordem variável de } \\
\text { itens durante o } \\
\text { treinamento. }\end{array}$ & $\begin{array}{l}\text { Amostra continha } 3 \\
\text { pacientes com } \\
\text { diagnóstico de APPvs }\end{array}$ & $\begin{array}{l}\text { Para o treinamento era apresentado } \\
\text { uma página com desenho de um } \\
\text { objeto, que se tentava nomear. Se } \\
\text { não conseguisem nomear, era } \\
\text { convidados a virar a página, onde o } \\
\text { mesmo objeto estava com seu nome } \\
\text { escrito abaixo. Após o período de } \\
\text { treinamento foram reavaliados em } \\
\text { uma semana, quatro meses e } 7 \\
\text { meses. }\end{array}$ \\
\hline & $\begin{array}{l}\text { Analisar a capacidade } \\
\text { de generalização do } \\
\text { aprendizado. }\end{array}$ & $\begin{array}{l}\text { Amostra com } 2 \\
\text { pacientes com APPvs }\end{array}$ & $\begin{array}{l}\text { Na linha de base e sessões de } \\
\text { avaliação incluíram duas fotos } \\
\text { adicionais para cada item que } \\
\text { investigou o comportamento da } \\
\text { generalização. Uma era item novo e } \\
\text { outro era semântica e visualmente } \\
\text { semelhante. }\end{array}$ \\
\hline $\begin{array}{l}\text { Croot, Taylor, } \\
\text { Abel, Jones, } \\
\text { Krein e } \\
\text { Hameister }{ }^{27}\end{array}$ & $\begin{array}{l}\text { Teve como objetivo a } \\
\text { recuperação lexical } \\
\text { para pessoas com } \\
\text { anomia. }\end{array}$ & $\begin{array}{l}2 \text { indivíduos com App } \\
\text { (1 variante logopênica } \\
\text { e } 1 \text { Não-fluente) de } \\
54 \text { e } 80 \text { anos }\end{array}$ & $\begin{array}{l}\text { As imagens para nomeação foram } \\
\text { apresentadas na tela do computador } \\
\text { acompanhadas de etiquetas para } \\
\text { leitura, escuta da palavra e repetição } \\
\text { da palavra alvo. }\end{array}$ \\
\hline
\end{tabular}


Tabela 2. Resumo dos resultados e conclusão dos artigos.

\begin{tabular}{|c|c|c|}
\hline Autores & Resultado & Conclusão \\
\hline $\begin{array}{l}\text { Beales } \\
\text { Whitworth } \\
\text { e } \\
\text { Panegyres }^{23}\end{array}$ & $\begin{array}{l}\text { Os resultados indicaram melhorias } \\
\text { significativas de itens tratados e ainda, } \\
\text { melhoria generalizada em palavras não- } \\
\text { tratadas. A melhora manteve-se após } 4 \\
\text { semanas da intervenção. }\end{array}$ & $\begin{array}{l}\text { o estudo fornece evidências de que indivíduos } \\
\text { com APP podem apresentar um ganho através } \\
\text { de uma intervenção léxica. Como também, } \\
\text { confirma a capacidade desses indivíduos de } \\
\text { aprender e/ou reaprender palavras tratadas e } \\
\text { não tratadas. }\end{array}$ \\
\hline $\begin{array}{l}\text { Savage, } \\
\text { Piguet e } \\
\text { Hodges }^{24}\end{array}$ & $\begin{array}{l}\text { Os resultados indicaram que todos os } \\
\text { participantes mostraram melhorias } \\
\text { significantes na nomeação de imagens } \\
\text { treinadas e da generalização. Mas para } \\
\text { itens não treinados não foi observado a } \\
\text { generalização. }\end{array}$ & $\begin{array}{l}\text { A utlização de tarefas ecológicas proporcionam } \\
\text { ganhos com valores reias para os pacientes }\end{array}$ \\
\hline $\begin{array}{l}\text { Savage, } \\
\text { Piguet e } \\
\text { Hodges }^{25}\end{array}$ & $\begin{array}{l}\text { Foram observadas reduções no } \\
\text { desempenho da nomeação na maioria } \\
\text { dos pacientes quando a prática foi } \\
\text { retirada. Apesar desses declínios } \\
\text { iniciais, pelo menos } 80 \% \text { das palavras } \\
\text { produzidas corretamente na pós- } \\
\text { avaliação imediata ainda eram } \\
\text { conhecidas seis meses depois, } \\
\text { independentemente da gravidade da } \\
\text { doença do participante (leve, moderada } \\
\text { ou grave). }\end{array}$ & $\begin{array}{l}\text { O período de manutenção é fundamental para } \\
\text { sustentar palavras durante um período de } 6 \\
\text { meses e pode ser através de formas menos } \\
\text { intensas de treinamento. }\end{array}$ \\
\hline $\begin{array}{l}\text { Hoffman, } \\
\text { Clarke, } \\
\text { Jones e } \\
\text { Noonan }\end{array}$ & $\begin{array}{l}\text { 1) O principal achado do foi a melhora } \\
\text { significante na retenção do vocabulário } \\
\text { de itens treinados. } \\
\text { 2) A exposição a múltiplos exemplos de } \\
\text { cada item durante o processo de } \\
\text { reaprendizagem melhorara a capacidade } \\
\text { de generalizar o vocabulário aprendido } \\
\text { para novos exemplos. }\end{array}$ & $\begin{array}{l}\text { As experiências de aprendizado mais variáveis } \\
\text { beneficiam os pacientes porque eles mudam a } \\
\text { estratégia do aprendizado e acabam ativando o } \\
\text { sistema semântico. O sucesso de portanto, essa } \\
\text { abordagem depende criticamente da } \\
\text { integridade das representações semânticas dos } \\
\text { itens sendo treinados. Pacientes com } \\
\text { comprometimento da nomeação no contexto de } \\
\text { déficits de compreensão relativamente leves } \\
\text { provavelmente se beneficiarão dessa } \\
\text { abordagem, evitando as consequências } \\
\text { negativas do excesso de avaliação. }\end{array}$ \\
\hline $\begin{array}{l}\text { Croot, } \\
\text { Taylor, } \\
\text { Abel, Jones, } \\
\text { Krein e } \\
\text { Hameister }\end{array}$ & $\begin{array}{l}\text { Os resultados indicaram uma melhora } \\
\text { significante nas habilidades de } \\
\text { nomeação para os itens tratados, se } \\
\text { mostrou capaz de generalização pra } \\
\text { imagens diferentes dos itens tratados. } \\
\text { Porém, não houve generalização para } \\
\text { itens não tratados. }\end{array}$ & $\begin{array}{l}\text { O estudo evidência melhora na amônia em } \\
\text { indivíduos APP. Essa melhora importante para } \\
\text { capacidade de conversação no dia a dia. }\end{array}$ \\
\hline
\end{tabular}

\section{O estudo de Beales et a/23 teve como objetivo analisar}

\section{os efeitos da intervenção em recuperação léxica auto}

\section{sinalizada entre as classes de palavras (substantivos, verbos}

e adjetivos). Para o estudo participaram 4 pacientes com 0

diagnóstico clínico de APP, idade entre 52-77 anos, três

sugestivos da APPvs e um APPvl. Na Figura 1, podemos

observar o desenho do estudo. 


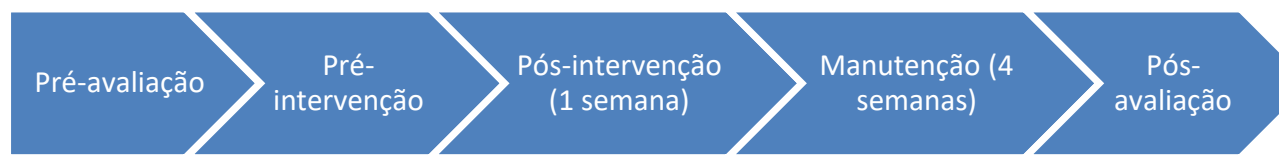

A intervenção integrou níveis semânticos, fonológicos e ortográficos de produção linguística baseados na memória autobiográfica. Foram realizadas oito sessões de terapia quinzenal de 90 minutos ao longo de 4 semanas na casa dos participantes. A abordagem de intervenção implementada para todos os participantes teve como objetivo desenvolver uma estratégia de recuperação léxica auto sinalizada. Nas atividades autobiográficas eram dados comandos (por exemplo, para o modelo "nome'"; "você tem um desses?"), instruções semânticas (por exemplo, para o modelo "substantivo'"; "De que categoria ela pertence?'), instruções ortográficas (por exemplo, "com que letra a palavra começa?") e instruções fonológicas (por exemplo, "com que som a palavra começa?"). Foram comparados resultados da pré-avaliação, da avaliação pós-intervenção e da avaliação após 4 semanas da intervenção.

Os resultados indicaram melhorias significantes na nomenclatura de itens tratados e em todas as classes de palavras, e ainda, melhoria generalizada em palavras não tratadas. A melhora manteve-se após 4 semanas da intervenção. Portanto, o estudo fornece evidências de que 
indivíduos com APP podem apresentar um ganho através de uma intervenção léxica. Como também, confirma a capacidade desses indivíduos de aprender e/ou reaprender palavras tratadas e não tratadas.

Savage et $a^{24}$ desenvolveu um treinamento cognitivo on-line para reaprender os nomes de objetos, com uma amostra de cinco pacientes de idade entre 56 e 71 anos e diagnóstico de APPvs. Juntamente com a família de cada participante, foi elaborado o programa individual com fotografias digitais de itens do cotidiano (alimentos, vestuário e eletrodomésticos), variando de 75 a 100 itens selecionados e distribuídos em três grupos (frequência das palavras, familiaridade e desempenho de nomeação). Foram utilizados de 10 a 15 objetos que poderiam ser nomeados corretamente para incentivar e diminuir medo ao erro. A primeira fase consistiu na construção da linha de base (préavaliação), variando de 4 a 15 avaliações antes do treinamento por paciente.

A intervenção foi realizada pela internet com uso do computador dos participantes e softwares personalizados. As atividades envolviam o emparelhamento de fotografias com seu respectivo rótulo (apresentado tanto em formato escrito como através de uma gravação de áudio da palavra falada). Foram propostas duas listas, cada uma foi aplicada por 5 dias/semana durante 4 semanas e levava em torno 30 minutos. No final de cada semana foi aplicado um teste de nomeação on-line. As imagens eram apresentadas uma de 
cada vez em ordem aleatória, com o participante tendo 10 segundos para responder.

Após oito semanas foi realizada outra avaliação presencial. Para avaliar o resultado do programa, foram comparados a linha de base, as avaliações on-line semanais, a avaliação pós-treinamento e o efeito de generalização.

Para as avaliações pré e pós foram criados pequenos vídeos (2 a $3 \mathrm{~min}$ ) de diferentes cenas domésticas, envolvendo itens treinados e não treinados. Após assistirem os vídeos, pedia-se ao paciente para descrevê-los. Para avaliar a compreensão das palavras foi solicitado que completassem frases utilizando itens treinados.

Os resultados indicaram que todos os participantes mostraram melhoras significativas na nomeação de imagens treinadas pós-intervenção. Observaram aumento na recuperação de palavras para itens treinados na atividade de descrição do vídeo, mas não ocorreu generalização para itens não treinados. Foi positivo a utilização de tarefas ecológicas (utilizando a nomeação de objetos do cotidiano) que proporcionam ganhos com valores reias para os pacientes.

Em 2015, Savage et a/25 realizaram uma pesquisa com objetivo de treinar e monitorar o desempenho de nomeação em uma série de pacientes APPvs, examinando a manutenção independente e assistida. Devido à variedade das diferenças individuais entre os pacientes, um objetivo secundário era identificar variáveis clínicas pré-tratamento associadas aos resultados da intervenção (tanto para o grau 
de aprendizado alcançado quanto para o desempenho de manutenção). A identificação de tais variáveis pode auxiliar na previsão da abordagem necessária para o sucesso do paciente em estudos futuros.

A amostra foi composta por indivíduos de 50 a 71 anos, com diagnóstico APPvs, com padrão típico de atrofia do lobo temporal anterior com perda de volume maior a direita em sete pacientes e à esquerda em dois participantes. Foi realizado um estudo de linha de base individual com três listas de palavras (itens do cotidiano: como alimentos ou roupas, com categorias adicionais, como plantas e instrumentos musicais) para cada paciente em um período de 3 a 4 semanas, após o qual os participantes foram treinados em sua primeira lista de palavras por 4 semanas. E, mais uma lista foi treinada por mais 4 semanas. Durante - mesmo período, uma lista de controle separada permaneceu sem tratamento. $O$ procedimento do treinamento envolveu o emparelhamento de uma fotografia de cada objeto, com uma palavra escrita e em áudio, no computador. Os participantes foram incentivados a olhar, ouvir e repetir a palavra, conforme ela estava sendo apresentada.

Os participantes foram reavaliados no final de cada semana de terapia e o desempenho foi monitorado por mais 6 meses, primeiro em intervalos semanais (nos primeiros 1 a 3 meses) e depois em intervalos mensais. Após intervenção, o treinamento foi reintroduzido quando foram observadas evidências de declínio. 
Como esperado, foram observadas reduções no desempenho da nomeação na maioria dos pacientes quando a prática foi retirada. Apesar desses declínios iniciais, pelo menos $80 \%$ das palavras produzidas corretamente na pósavaliação imediata ainda eram conhecidas seis meses depois, independentemente da gravidade da doença do participante (leve, moderada ou grave). Esse nível de desempenho ao longo do tempo, no entanto, foi alcançado usando uma variedade de abordagens, desde manutenção independente até revisão contínua. É importante ressaltar que, embora a prática intensa tenha auxiliado no rápido reaprendizado inicial das palavras, os horários de prática menos intensos foram capazes de sustentar a maioria das palavras ao longo do tempo

A pesquisa realizada por Hoffman et $a^{26}$ tinha como objetivo investigar a reaprendizagem para melhorar a generalização, fornecendo um vocabulário que os pacientes podessam aplicar em suas vidas cotidianas. Esta pesquisa foi realizada em duas partes e utilizou uma amostra de três pacientes com diagnóstico de APPvs, com idade entre 56 e 63 anos.

Primeiramente analisou o efeito de ordem variável de itens durante o treinamento. $O$ desenho do estudo um está resumido na Figura 2. Inicialmente foi realizada uma avaliação em duas sessões onde os pacientes teriam que combinar 120 figuras à palavra correspondente. Os resultados obtidos foram utilizados para criar três conjuntos de 25 itens os quais não conseguiam corresponder 
corretamente nas duas sessões. Cada conjunto foi dividido em: ordem fixa, ordem variável ou controle. Na etapa seguinte foi realizado o treinamento de reaprendizagem com os conjuntos de ordem fixa e variável. Os itens do conjunto controle não foram apresentados durante o treinamento.

Figura 2. Desenho do estudo de Hoffman et $a^{26}$.

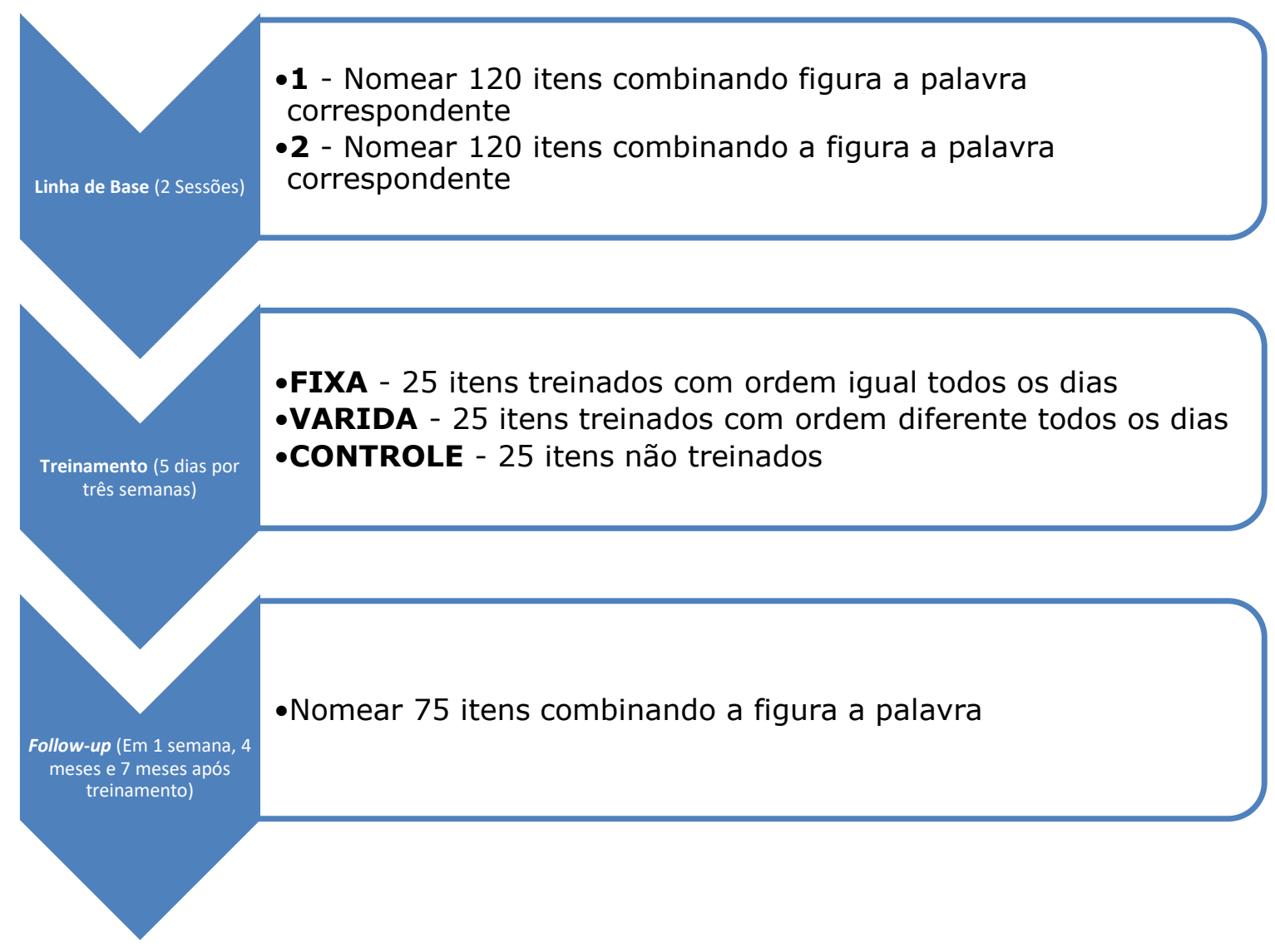

Para o treinamento de reaprendizagem, cada paciente tinha pasta com material. Era apresentado uma página com desenho de um objeto, que tentava nomear. Se não conseguisse nomear, era convidado a virar a página, onde o mesmo objeto estava com seu nome escrito abaixo. Era 
solicitado para lerem três vezes o nome em voz alta, caso ocorressem erros, estes eram corrigidos pelo investigador. Eram apresentados os dois conjuntos diariamente, de segunda à sexta-feira por três semanas, por cerca de 20 minutos por dia. Após o período de treinamento foram reavaliados com combinação de imagem à palavra correspondente de 75 itens após uma semana, quatro meses e sete meses.

O principal achado do estudo 1 foi a melhora significante na retenção do vocabulário de itens treinados. Com estratégia de ordem variável ocorreu aprimoramento do conhecimento.

No estudo 2, os pesquisadores aumentaram a variabilidade dos itens, com série diferentes de exemplos, com objetivo de analisar a capacidade de generalização do aprendizado. Na figura 3 podemos observar a descrição das atividades. O treinamento foi dividido em dois grupos de tarefas, um deles foi exposto com exemplos múltiplos (três variedades diferentes de cada objeto) através de fotografias. Outro grupo, consistiu em exemplo único (apenas uma variedade de cada objeto). Nos dois grupos foram expostos 25 itens, mas com os diferenciais de exemplo único e outro múltiplo. Para corresponder ao valor total do treinamento nos dois grupos, esse único exemplo foi apresentado três vezes em cada sessão. Os pacientes também completaram uma avaliação de base e avaliações de acompanhamento em 1 semana, 1 mês, 4 meses e 7 meses. Na linha de base e sessões de avaliação incluíram duas fotos adicionais para 
cada item que investigou o comportamento da generalização. Uma delas era um novo exemplo do item não usado durante o treinamento e o outro era uma folha que era semântica e visualmente semelhante ao item treinado.

Figura 3. Segunda parte do desenho do estudo de Hoffman et a ${ }^{26}$.

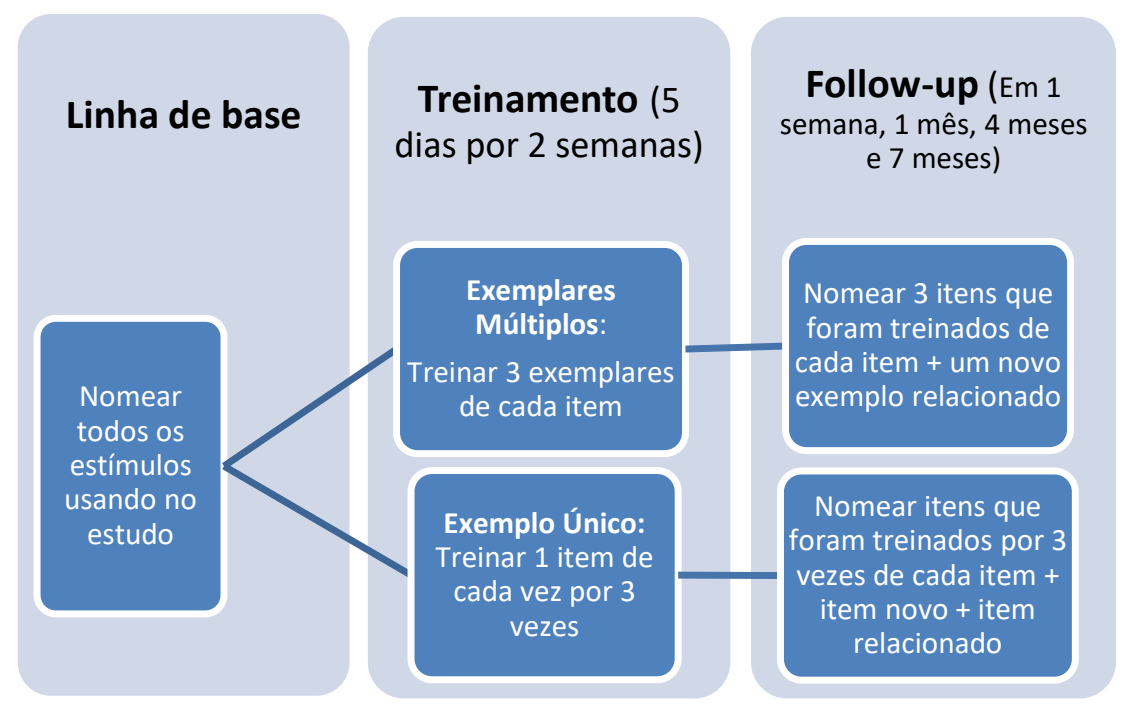

O principal achado do estudo 2 foi que a exposição a múltiplos exemplos de cada item durante o processo de reaprendizagem melhora a capacidade de generalizar o vocabulário aprendido para novos exemplos. No entanto, esses ganhos tiveram um custo: tornou-se mais provável usar erroneamente as palavras aprendidas para nomear outros objetos que eram semântica e visualmente semelhantes aos usados no treinamento. 
O estudo de Croot et $a^{27}$ utilizou uma amostra de dois indivíduos de 54 e 80 anos com diagnóstico APP, o primeiro com variante logopênica e o segundo não-fluente. Teve como objetivo a recuperação lexical para pessoas com anomia. A intervenção foi realizada em domicílio através de imagens para nomeação apresentadas na tela do computador acompanhadas de etiquetas para leitura, escuta da palavra e repetição da palavra alvo. As palavras foram préselecionadas em dois tópicos relevantes para uma conversação e foram avaliadas durante uma entrevista estruturada como medida basal (pré-teste) e comparadas após tratamento.

As atividades semânticas incluídas nos estudos eram de recuperação, nomeação e elaboração semântica, através de classificação de imagens, vídeos ou palavras. Os resultados indicaram uma melhora significante nas habilidades de nomeação para os itens tratados. Os indivíduos se mostraram capazes de generalização para imagens diferentes dos itens tratados. Porém, não houve generalização para itens não tratados. São necessárias mais pesquisas para analisar o potencial e limites da reabilitação de anomia durante uma conversa.

\section{DISCUSSÃO}

O objetivo do presente artigo foi analisar as técnicas ecológicas de reabilitação neuropsicológica e sua efetividade no tratamento de indivíduos com APP através da revisão sistemática de literatura. 
Os resultados gerais sugerem que as intervenções de reaprendizagem da nomeação para itens conhecidos através da exposição de imagens com seu respectivo nome escrito, áudio da palavra e sua repetição resultaram numa melhora significativa para esses pacientes. Observou-se que a reabilitação neuropsicológica baseada no cotidiano do paciente é um instrumento importante para tratamento de pacientes com APP.

Os estudos de Beales et $a /^{23}$, Savage et $a /^{24}$, Croot et al ${ }^{27}$ e Hoffman et al ${ }^{26}$ evidenciaram um ganho significante de generalização para itens tratados. Apenas o estudo de Beales et $a^{23}$ conseguiu demonstrar generalização para itens não tratados. Um dos motivos pode ter sido porque sua a pesquisa utilizou técnicas de estimulação cognitiva com base semântica, além da nomeação (escrita e auditiva) e os itens selecionados foram baseados autobiografia. Assim, quando os itens trabalhados fazem parte da memória semântica, estes possuem maior probabilidade de serem retidos ${ }^{28}$. O que evidencia que técnicas ecológicas ou baseadas no interesse do paciente resultam numa maior capacidade de generalização de itens tratados e não-tratados ${ }^{29}$.

Uma outra questão importante a considerar quanto a capacidade de ganho na generalização quando comparamos estudos de Beales et $a^{23}$ e Croot et a/27 são os subtipos de APP das amostras. No estudo de Beales et a/23 a amostra foi composta por três pacientes com APPvs e um com APPvl no qual o primeiro subtipo tem como característica a anomia e no segundo dificuldade de repetição e nomeação de 
confronto, os quais foram trabalhados nas intervenções. Enquanto estudo de Croot et a/27 compôs sua amostra com um paciente APPvl e outros APPvnf no qual tem como característica agramatismo e apraxia da fala. Assim apenas $50 \%$ da sua amostra tinha como principal deficiência dos pontos tratados. Talvez, o paciente APPvnf teria melhores resultados com desenvolvimento de tarefas baseadas na articulação das palavras.

Apenas dois estudos avaliaram a capacidade de manutenção, indicando que a reaprendizagem se manteve por um período de até 6 (Savage et $a^{25}$ ) e 7 meses (Hoffman et $a^{26}$ ) após as intervenções. Estudo de Savage et a/25 analisou o período de manutenção e demonstrou que a manutenção contínua, através de formas menos intensas de treinamento, pode ajudar os pacientes com APPvs a sustentar palavras durante um período mais longo. Resultado muito importante para ser considerado na pesquisa em pacientes com APP, considerando que estes estão em um processo neurodegenerativo ${ }^{20}$.

O estudo de Hoffman et a/26 apresentou múltiplos exemplos de cada item de objeto selecionado desencadeando um maior número de erros nas palavras aprendidas quando apresentavam semelhança semântica ou visual. Utilizar exemplos diferentes pode não ter fortalecido a associação entre a imagem e seu nome, sugerindo uma melhora apenas na recuperação lexical ${ }^{29}$.

Ainda são necessárias novas pesquisas que considerem amostragem randomizada, com maior número de indivíduos 
e que comparem a eficiência do tratamento em relação à variante de APP. Dessa forma, teríamos mais dados que tornasse possível elaborar protocolos de tratamento com intervenções adequadas para cada variante, uma vez que cada variante apresenta alterações específicas nos domínios linguísticos na produção de fala, repetição, compreensão de uma única palavra e sintaxe, nomeação de confrontos, conhecimento semântico e leitura/ortografia ${ }^{6}$.

Estudos de reabilitação mais estruturados visando compensar as deficiências de cada variante de APP e manter o funcionamento da vida diária seriam benéficos ${ }^{30} \mathrm{com}$ técnicas que se concentrassem nas características dos objetos (uso, localização e aparência) ${ }^{31}$. Como também, que considerassem a capacidade de gerenalização para itens tratados e não-tratados e capacidade de manutenção.

\section{CONCLUSÃO}

O tratamento e intervenções cognitivo-linguísticas direcionadas principalmente para nomeação e através de terapia semântica, ortográfica e fonológica podem beneficiar muito os pacientes com APP, ao contribuir para proteção ou melhora da função linguística.

Porém ainda são necessárias novas pesquisas que considerem amostragem randomizada, com maior número de indivíduos e que comparem a eficiência do tratamento em relação à variante de APP. Como também, incluir nos estudos a análise de generalização e manutenção dos resultados. 
Para que num futuro possam elaborar protocolos de tratamento com intervenções adequadas para cada variante.

\section{REFERÊNCIAS}

1.Gorno-Tempini ML, Tee BL. Primary progressive aphasia: a model for neurodegenerative disease. Curr Opin Neurol 2019;32:255-65. https:/doi.org/10.1097/WC0.0000000000000673

2. Warren JD, Rohrer JD, Schott JM, Fox NC, Hardy J, Rossor MN. Molecular nexopathies: a new paradigm of neurodegenerative disease. Trend Neurosci 2013;36:561-9. https://doi.org/ 10.1016/j.tins.2013.06.007

3.Utianski LR, Botha H, Martin PR, Schwarz CG, Duffy JR, Clark HM, et al. Clinical and Neuroimaging Characteristics of Clinically Unclassifiable Primary Progressive Aphasia. Brain Lang 2019;197:104676. https://doi.org/10.1016/j.bandl.2019.104676

4.Gorno-Tempini $M L$, Hillis $A E$, Weintraub $S$, Kertesz $A$, Mendez $\mathrm{M}$, Cappa SF, et al. Classification of primary progressive aphasia and its variants. Neurology 2011;76:1006-14.

http://doi.org/10.1212/WNL.0b013e31821103e6

5. Mesulam MM. Primary progressive aphasia: a language-based dementia. N Engl J Medicine 2003;349:1535-42. https://doi.org/10.1056/NEJMra022435

6. Mesulam MM, Weintraub S. Spectrum of primary progressive aphasia. Baillieres Clin Neurology 1992;1:583-609. https://pubmed.ncbi.nlm.nih.gov/1344204/

7. Hodges J, Patterson K, Oxbury S, Funnell E. Semantic dementia. Progressive fluent aphasia with temporal lobe atrophy. Brain 1992;115:1783-806. http://doi.org/10.1093/brain/115.6.1783

8.Snowden JS, Goulding PJ, Neary D. Semantic dementia: a form of circumscribed cerebral atrophy. Behav Neurol 1989;2:167-82. https://doi.org/10.1155/1989/124043

9.Gainotti G. Different patterns of famous people recognition disorders in patients with right and left anterior temporal lesions: a systematic review. Neuropsychol 2007;45:1591-607.

http://doi.org/10.1016/j.neuropsychologia.2006.12.013

10.Wilson BA, Winegardner J, van Heugten CM, Ownsworth $T$. Neuropsychological Rehabilitation The International Handbook. Inglaterra: Routledge, 2017. https://doi.org/10.4324/9781315629537

11. Marshall JC, Newcombe F. Patterns of paralexia: a psycholinguistic approach. J Psycholing Res 1973;3:175-99. https://doi.org/10.1007/BF01067101

12. Mesulam MM. Primary progressive aphasia. Ann Neurol 2001;49:425-32. https://doi.org/10.1002/ana.91 
13. Mesulam $M$, Wicklund A, Johnson N, Rogalski $E$, Léger GC, Rademaker $A$, et al. Alzheimer and frontotemporal pathology in subsets of primary progressive aphasia. Ann Neurol 2008;63:709-19. https://doi.org/10.1002/ana.21388

14.Grossman M, Mickanin J, Onishi K, Hughes E, D'Esposito M, Ding X $S$, et al. Progressive non-fluent aphasia: language, cognitive and PET measures contrasted with probable Alzheimer's disease. J Cogn Neurosci 1996;8:135-54. http://doi.org/10.1002/ana.10825 15.Gorno-Tempini ML, Dronkers NF, Rankin KP, Ogar JO, Phengrasamy $\mathrm{L}$, Rosen $\mathrm{HJ}$, et al. Cognition and anatomy in three variants of primary progressive aphasia. Ann Neurol 2004;55:335-46. http://doi.org/10.1002/ana.10825

16.Wilson SM, Brambati SM, Henry RG, Handwerber DA, Agosta F, Miller $\mathrm{BL}$, et al. The neural basis of surface dyslexia in semantic dementia. Brain

86. https:/doi.org/10.1093/brain/awn300

17. Rizzo AA, Schultheis M, Kerns KA, Mateer C. Analysis of assets for virtual reality applications in neuropsychology. Neuropsychol Rehab 2004;14:207-39. https://doi.org/10.3414/ME15-01-0050

18. Tippett DC, Hillis AE, Tsapkini K. Treatment of primary progressive aphasia. Curr Treat Options Neurol 2015;17:362. https://doi.org/ 10.1007/s11940-015-0362-5

19. Meyer AM, Tippett DC, Friedman RB. Prophylaxis and remediation of anomia in the semantic and logopenic variants of primary progressive aphasia. Neuropsychol Rehab 2018;28:352-68. https://doi.org/10.1080/09602011.2016.1148619

20.Cadório I, Lousada M, Martins P, Figueiredo D. Generalization and Maintenance of Treatment Gains in Primary Progressive Aphasia (PPA): A Systematic Review. Int J Lang Commun Disord 2017;52:543-60. http://doi.org/10.1111/1460-6984.12310

21. Meyer AM, Getz HR, Brennan D, Hu T, Friedman RB. Telerehabilitation of anomia in primary progressive aphasia. Aphasiology 2016;30:483-507. https://doi.org/10.1080/02687038.2015.1081142

22. Cotelli M, Manentia R, Ferrarib C, Gobbi E, Macis A, Stefano FC. Effectiveness of language training and non-invasive brain stimulation on oral and written naming performance in Primary Progressive Aphasia: A meta-analysis and systematic review. Capp Neurosci Biobehav Rev 2020;108:498-52. http://doi.org/10.1016/j.neubiorev.2019.12.003

23.Beales A, Whitworth C, Panegyres PK. Exploring generalization processes following lexical retrieval intervention in primary progressive aphasia. Inter J Speech Lang Pathol 2016;18:299-314. https://doi.org/10.3109/17549507.2016.1151936

24.Savage SA, Piguet O, Hodges JR. Giving Words New Life: Generalization of Word Retraining Outcomes in Semantic Dementia. J Alzheimers Dis 2014;40:309-17. https://doi.org/10.3233/JAD-131826 
25.Savage SA, Piguet O, Hodges JR. Cognitive Intervention in Semantic Dementia: Maintaining Words Over Time. Alzheimer Dis Assoc Disord 2015;29:55-62. https://doi.org/10.1097/WAD.0000000000000053

26. Hoffman P, Clarke N, Jones RW, Noonan KA. Vocabulary Relearning in Semantic Dementia: Positive and Negative Consequences of Increasing Variability in the Learning Experience. J Neuropsychol $2015 ; 76: 240-53$.

http://doi.org/10.1016/j.neuropsychologia.2015.01.015

27. Croot K, Taylor C, Abel S, Jones K, Krein L, Hameister L, et al. Measuring gains in connected speech following treatment for word retrieval: a study with two participants with primary progressive aphasia. Journal Aphasiology: 2014;29:1265-1288. https://doi.org/10.1080/02687038.2014.975181

28.Jokel R, Graham NL, Rochon E, Leonard C. Word retrieval therapies in primary progressive aphasia. Aphasiology 2014;28:1038-68. https://doi.org/10.1080/02687038.2014.899306

29.Krajenbrink T, Croot K, Taylor-Rubin CN. Treatment for spoken and written word retrieval in the semantic variant of primary progressive aphasia. Neuropsychol Rehab 2020;30:915-47. https://doi.org/10.1080/09602011.2018.1518780

30.Carthery-Goulart MT, da Silveira AC, Machado, TH, Mansur LL, Parente MAMP, Senaha MLH. Nonpharmacological interventions for cognitive impairments following primary propressive aphasia: a systematic review of the literature. Dem Neuropsychol 2013;7:122-31. http://doi.org/10.1590/S1980-57642013DN70100018

31. Marshall CR, Hardy CJD, Volkmer A, Russell LL, Bond RL, Fletcher PD, et al. Primary progressive aphasia: a clinical approach. J Neurol 2018;265:1474-90. http://doi: 10.1007/s00415-018-8762-6 